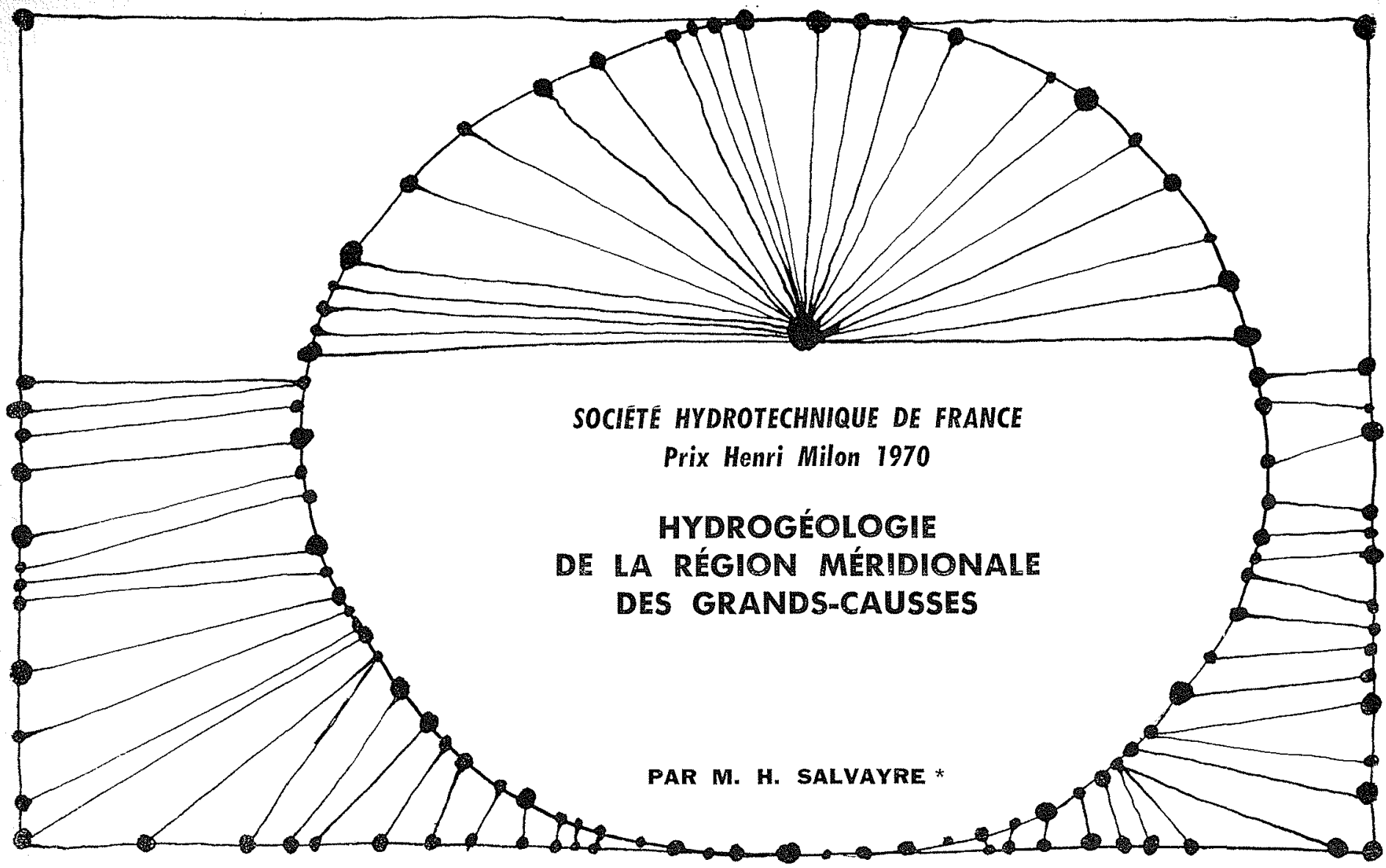

La présente étude est le résumé d'une thèse de doctorat d'Etat ("), préparée sous la direction de M. le Professeur H. Schoeller et de M. le Professeur J. Avias, et soutenue le 27 mai 1969 à Bordeaux.

\section{Présentation d'ensemble}

L'étude hydrogéologique qui fait l'objet de notre thèse, a été réalisée dans une double perspective:

- apporter une contribution à la reconnaissance des ressources en eau de la région méridionale des GrandsCausses et montrer les résultats que l'on peut obtenir par l'utilisation des moyens d'investigations classiques offerts par la climatologie, la potamologie, l'hydrogéologie et la géochimie, étroitement liés entre eux et appliqués au milieu calcaire:

- dégager de ces recherches quelques concepts de base, propres au mouvement de l'eau dans les calcaires, obtenus par l'observation directe sur le terrain ou résultant d'une recherche méthodologique mettant en ouvre les méthodes expérimentales ou analytiques propres à l'étude du déplacement de l'eau dans les terrains à perméabilité d'interstices et transposées au cas particulier du karst.

Le choix de la région méridionale des Grands Causses a été conditionné par plusieurs raisons (fig. 1).

La première est l'homogénéité stratigraphique et structurale des Causses qui réduit l'influence du milieu sur les formes de percolation d'écoulement et de rétention et les rend plus facilement accessibles à l'analyse critique ou à l'expérimentation.

La deuxième est l'importance de la masse karstifiable dans laquelle se développent un grand nombre de karsts

\footnotetext{
* Docteur ès-Sciences.

(*) Un exemplaire de cette thèse peut être consulté au secrétariat de la S.H.F., 199, rue de Grenelle, Paris $\left(7^{\circ}\right)$.
}

actifs et où s'observent une importante variété de formes d'alimentation et d'écoulement.

Notre travail comprend quatre grandes parties:

- une première partie est consacrée à l'étude climatologique de la région méridionale des Grands Causses. Dans ce chapitre, nous présentons et analysons les données pluviométriques et étudions leurs variations vis-à-vis de la topographie. Nous complétons ce chapitre par une étude des caractéristiques thermiques. Plus particulièrement par la recherche des valeurs de l'évapotranspiration, abordées par les différentes méthodes ou évaluées à partir de mesures directes:

- la seconde partie concerne l'hydrologie superficielle; elle comprend un exposé sur la morphologie du réseau hydrographique, l'étude du régime des débits faisant apparaître les débits moyens mensuels annuels et définissant le régime par le calcul des débits spécifiques, des coefficients de débit et de la hauteur de la lame d'eau écoulée. L'étude des crues comporte l'analyse et la séparation des composantes de l'hydrogramme précisées par le calcul des coefficients des différentes phases du tarissement et la valeur des volumes transités;

- la troisième partie intéresse l'hydrogéologie karstique proprement dite. Elle comprend deux chapitres:

Le premier est consacré à l'étude descriptive dies karsts régionaux.

Le second expose les résultats acquis à la suite de recherches méthodologiques sur les formes et les caractères des écoulements karstiques.

Le premier chapitre comporte: une introduction sur les caractères lithologiques et structuratux des niveaux d'écoulement liés aux faciès pétrographiques et chimiques, la distinction entre les différents systèmes hydrologiques et l'étude proprement dite des systèmes karstiques régionaux.

Le second chapitre se divise en six paragraphes relatifs au mouvement de l'eau dans le milieu calcaire et comprenant: les résultats des recherches par pompages sur les 


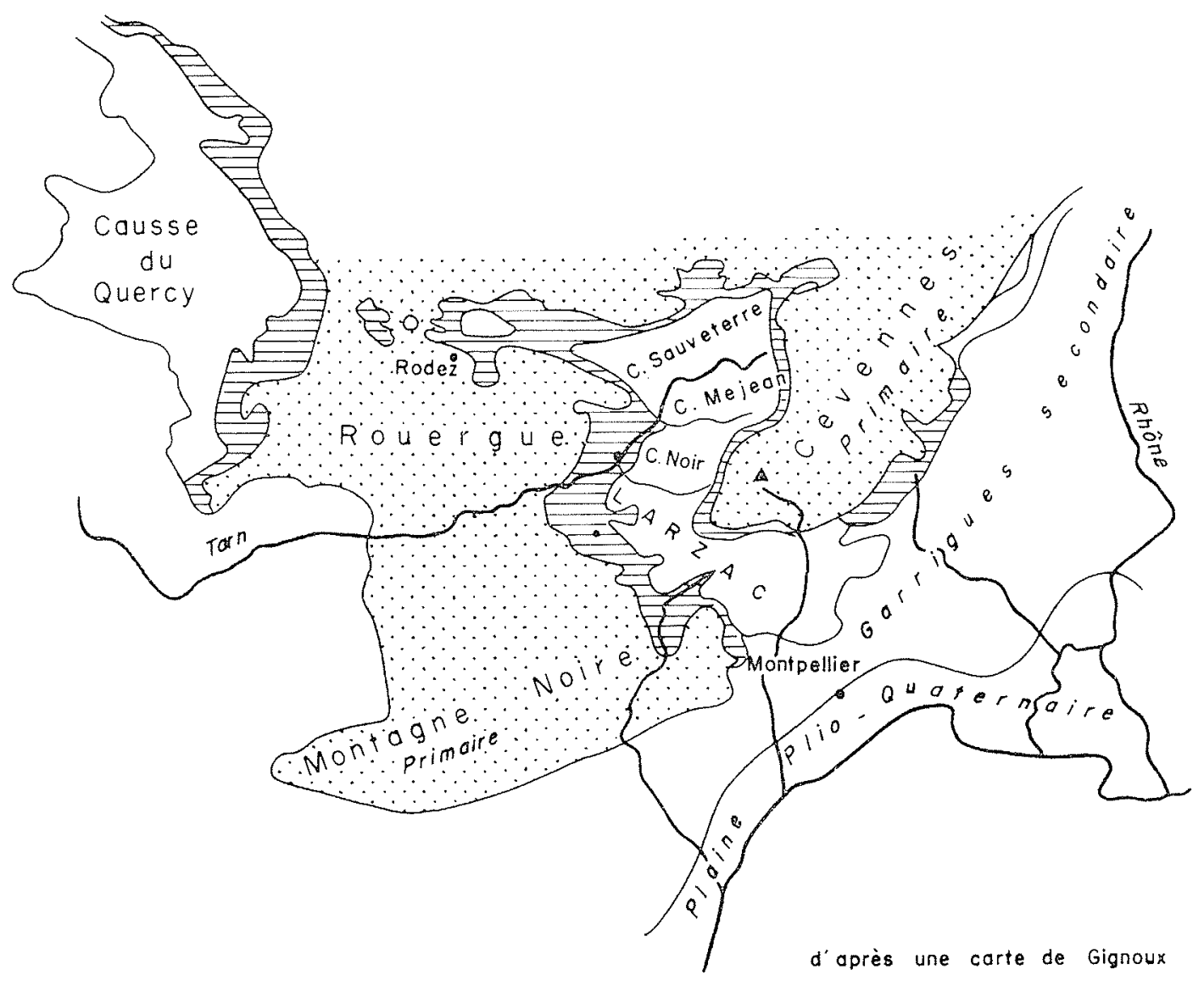

\section{SITUATION GEOGRAPHIQUE}

\section{DU LARZAC ET DE L'AVANT. CAUSSE}

Dessin

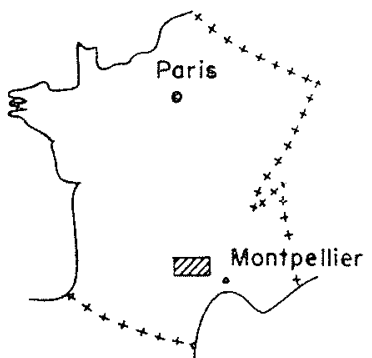

écoulements souterrains, l'étude comparée des hydrogrammes de tarissement aux résurgences; les observations sur l'influence des facteurs lithologiques et structuraux sur les écoulements; la recherche du bilan hydrologique par les méthodes de Thornthwaite, de Turc et le bilan du chlore; les valeurs des vitesses de circulation et du cavernement: la description des phénomènes d'extravasement et d'accumulation.

- nous avons réuni dans une quatrième partie les éléments propres à la chimie des eaux souterraines des Causses en mettant en évidence: quelques cas d'évolution chimique, les faciès hydrochimiques et la situation des Grands Causses par rapport aux karsts du sud de la France.

\section{Principaux résultats}

\section{Climakologie.}

Pluviométrie :

Placée entre trois massifs à forte pluviométrie : l'Aigoual (2 $038 \mathrm{~mm})$, le Lévézou (1 $200 \mathrm{~mm})$ et la Montagne Noire
(1 $300 \mathrm{~mm})$, la région méridionale des Grands Causses a ses modules pluviométriques compris entre $900 \mathrm{~mm}$ et $700 \mathrm{~mm}$ (fig. 2).

On observe des creux pluviométriques dans les vallées est-ouest (Millau : $673 \mathrm{~mm}$ ), des maximums dans les vallées nord-sud (Vis : $1200 \mathrm{~mm}$ ); on note des maximums en bordure du Causse ( $925 \mathrm{~mm}$ au Caylar) des minimums au centre $(765 \mathrm{~mm}$ à la Blaquèrerie et à larrière $(752 \mathrm{~mm}$ à SaintAffrique) (fig. 3).

Sur le Causse, les isohyètes sont indépendantes des courbes de niveaux. Les rapports pluviométriques maximum/minimum sont compris entre 3 et 5 al sud du Larzac, entre 1,4 et 2 au nord.

Juillet et février sont les mois les plus secs de l'année, octobre le mois le plus humide. Les courbes des hauteurs des précipitations classées soulignent par ailleurs la régularisation de la répartition des pluies lorsque l'on va du sud au nord des Causses, et indiquent ainsi le passage gradusl du climat méditerranéen au climat océanique, (fig. 4). 


\section{TEMPÉRATURES.}

La température moyenne annuelle est élevée: au Caylar elle est de $10,3^{\circ}$, à Millau de $11,5^{\circ}$, les minimums sont pourtant très marqués sur le plateau où il n'est pas rare de voir des températures inférieures à $-10^{\circ}$.

\section{EVAPOTRANSPIRATION.}

Les valeurs de l'évapotranspiration (formule de Turc ou de Thornthwaite) placent la région méridionale des Grands Causses dans une zone comprise entre $500 \mathrm{~mm}$ et $600 \mathrm{~mm}$.

Les mesures directes de l'évaporation effectuées sur l'Avant-Causse et le Causse, entre 1967 et 1968, donnent les valeurs suivantes:

\begin{tabular}{|c|c|c|}
\hline Statron & $\begin{array}{c}\text { Piche } \\
(\mathrm{mm})\end{array}$ & $\begin{array}{c}\text { BaC Colorado } \\
(\mathrm{mm})\end{array}$ \\
\cline { 2 - 3 } & $\begin{array}{c}82,6 \text { (oct.) } \\
\text { Avant-Causse }\end{array}$ & 62 (oct.) \\
(Soulobres). & 237,7 (juin) & 209,6 (juin) \\
Causse & 61,1 (oct.) & 38 (oct.) \\
(Cazejourdes) & 133,2 (août) & 140 (juin) \\
\hline
\end{tabular}

Cette étude montre la diversité des valeurs climatologiques sur une région d'aspect homogène, elle confirme l'intérêt des études climatologiques fines lorsque l'on désire connaître avec exactitude les éléments du bilan hydrolo gique.

\section{Hydrologie superíicielle.}

LE RÉSEAU HYDROGRAPHIOUE.

Sur le plan potamologique, nous avons tout d'abord étudié le réseau hydrographique en relation avec le milieu géologique et distingué les rivières autochtones du Causse : la Sorgues, le Cernon, la Vis, le cours amont de l'Orb et les rivières allogènes: le Tarn, la Dourbie, la Jonte et le Dourdou. Pour chacune d'entre elles, nous avons recherché les caractéristiques principales du bassin : surface, longueur, profil, débits d'étiages et de crues.

RÉGIME DES DÉBITS.

L'étude du régime des débits nous a montré que toutes ces rivières étaient caractérisées par de hautes eaux en mars et décembre, de basses eaux aux mois de juillet et août. Les mois d'hiver et de printemps sont déterminants pour le régime. L'automne malgré ses excès pluviométriques ne parvient pas à combler le déficit qui peut résulter d'une faible alimentation d'hiver ou de printemps.

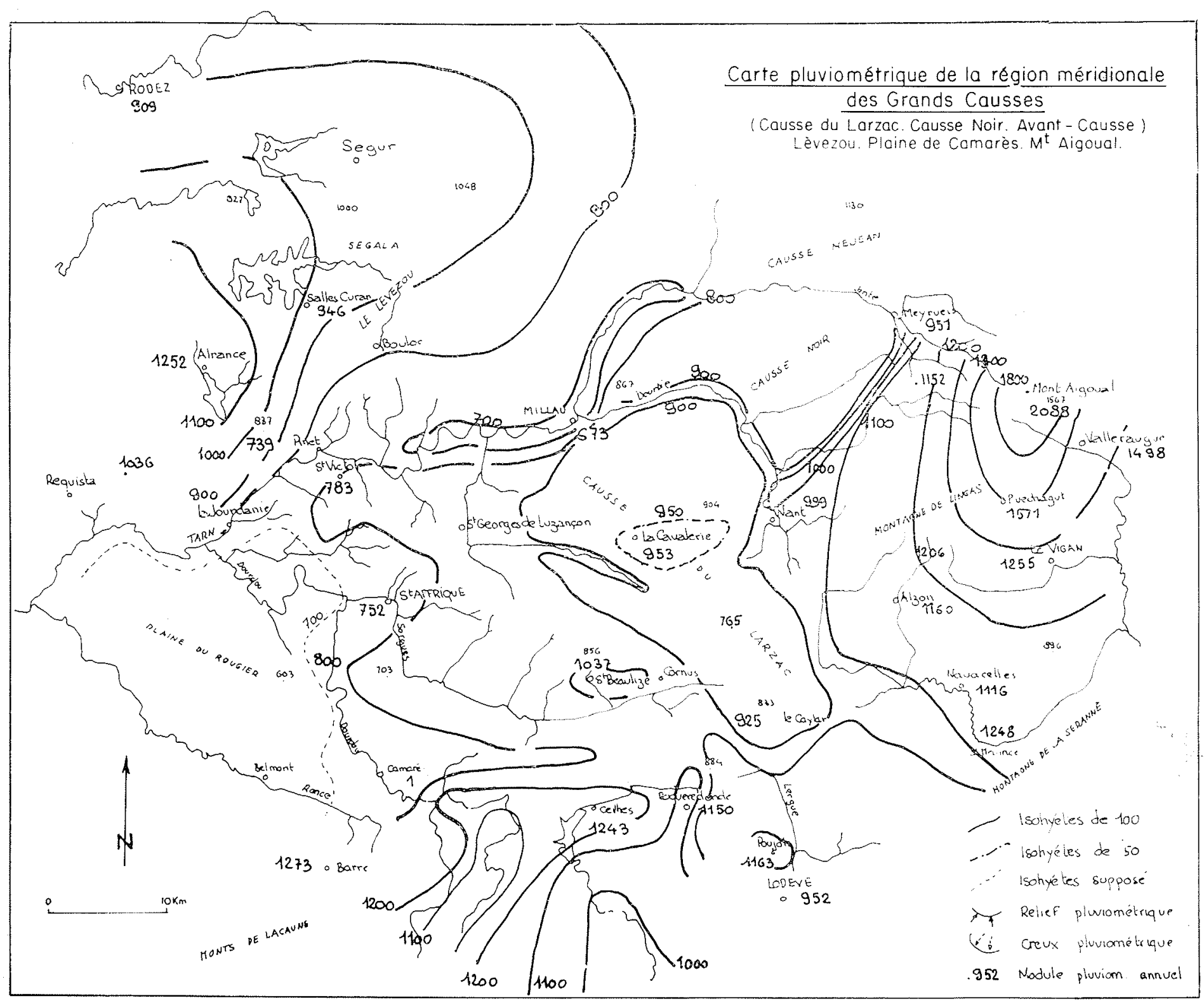




\section{H. SALVAYRe}

Les courbes des débits caractéristiques nous indiquent que les rivières ont un étiage tonjours supérieur ou égal à $1 \mathrm{~m}^{3} / \mathrm{s}$. Les débits spécifiques sont compris entre $45,71 / \mathrm{s} / \mathrm{km}^{2}$ pour la Dourbie et $14,81 / \mathrm{s} / \mathrm{km}^{2}$ pour la Vis (fig. 5 et 6 ). Le coefficient de débit varie entre le mois le plus humide et le mois le plus sec de 2 à 0,2 (fig. 7).

Le régime des rivières du Causse peut être défini comme un régime de type pluvionival méditerranéen à deux maximums d'amplitude (automne et printemps) et un étiage principal (été).

\section{LES CRUES.}

Les crues se répartissent en crues de printemps et d'automne, celles d'automne sont les plus fréquentes, celles du printemps se font essentiellement sentir dans les bassins du Dourdou et de la Sorgues. Les crues d'automne sont les plus violentes, elles se caractérisent par des temps de montée très courts de 2 à $6 \mathrm{~h}$, la pointe de l'hydrogramme marque un palier qui peut durer de 2 à $4 \mathrm{~h}$, la décrue est rapide : $48 \mathrm{~h}$ pour la Dourbie, $78 \mathrm{~h}$ pour le Tarn.

Les crues de la Dourbie sont particulièrement violentes et rapides, l'analyse de la genèse de la crue du 31 octobre 1963, nous a montré que les crues de la rivière prennent naissance dans son bassin supérieur à forte déclivité, imperméable et à pluviosité élevée.

L'analyse de la crue de la Sorgues du 2 octobre 1964 nous a également montré que la genèse des fortes crues qui affectent parfois les rivières de l'Avant-Causse, est liée à une trombe d'eau très localisée tombant sur le bassin moyen de la rivière (généralement non calcaire), dans le cas contraire, la masse calcaire provoque l'étalement de la crue.

\section{LES COMPOSANTES DES HYDROGRAMMES.}

Pour l'ensemble des rivières $\alpha_{1}$ n'est pas caractéristique, il est le plus petit pour la Vis $\left(0,5>\alpha_{1}>0,2\right)$ de l'ordre de 1,3 ailleurs. $\alpha_{2}$ est toujours de l'odre de $10^{-1}$, les valeurs les plus élevées sont celles du Tarn: $\alpha_{2}=0,82$, les plus faibles sont celles de la Vis : $0,03<\alpha_{2}<0,14$.

$\alpha_{3}$ est plus représentatif; on peut admettre que pour les rivières à bassin calcaire dominant on a $0,010>\alpha_{3}$ $>0,002$; pour les rivières à bassin calcaire important, mais non dominant, on a $0,09>\alpha_{3}>0,04$.

L'écoulement souterrain libère le volume maximal d'eau, la valeur ainsi transitée est de $93 \%$ à $84 \%$ pour la Vis, de $93 \%$ à $55 \%$ pour l'Orb et la Lergue et d'une façon générale pour les rivières de l'Avant-Causse.

Pour le Tarn et la Dourbie, l'écoulement intermédiaire garde une valeur importante pouvant atteindre $50 \%$. Le volume de l'écoulement du ruissellement varie de $22 \%$ à $0,3 \%$ pour les rivières à bassin calcaire dominant et de $11 \%$ à $25 \%$ pour les autres.

La forte valeur du volume transité par la dernière phase du tarissement explique la pérennité des rivières du Causse
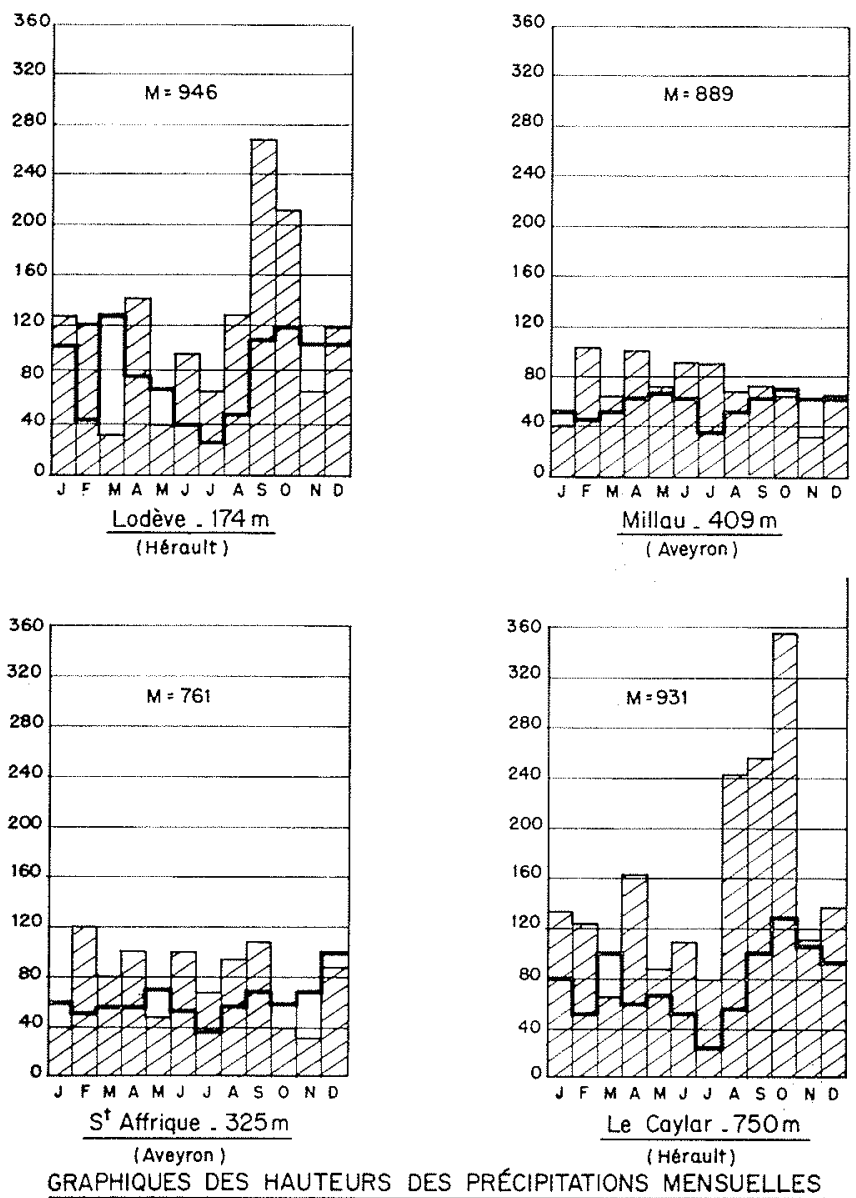

GRAPHIQUES DES HAUTEURS DES PRECIPITATIONS MENSUELLES

- Moyenne des pluies $T$ Pluie on 1963

$M=$ module on $\mathrm{mm}$

3/ Graphique des hauteurs des précipitations mensuelles à quelques stations du Causse.

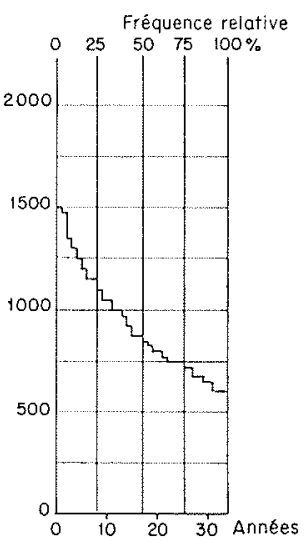

Modules pluviométriques annuels classés au Caylar de 1928-1963

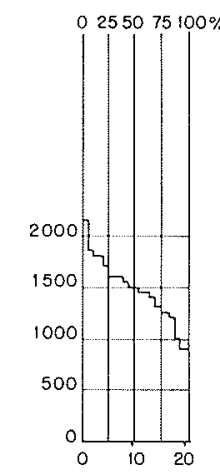

Modules pluviométriques annuels classés a St Affrique 1943-1963

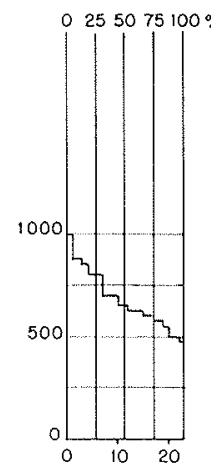

Modules pluviométriques annuels classês à Millau 1941-1963

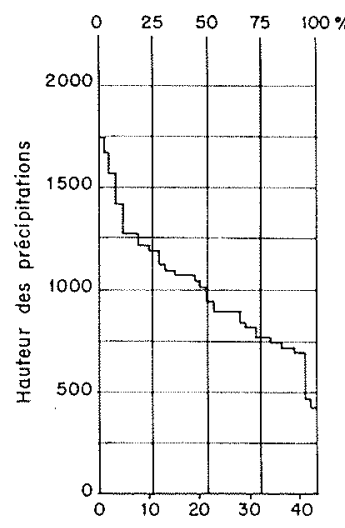

Modules pluviométriques annuels classés a Lodève de $1921-1963$

4/ Modules pluviométriques classés pour quelques stations des Causses. 

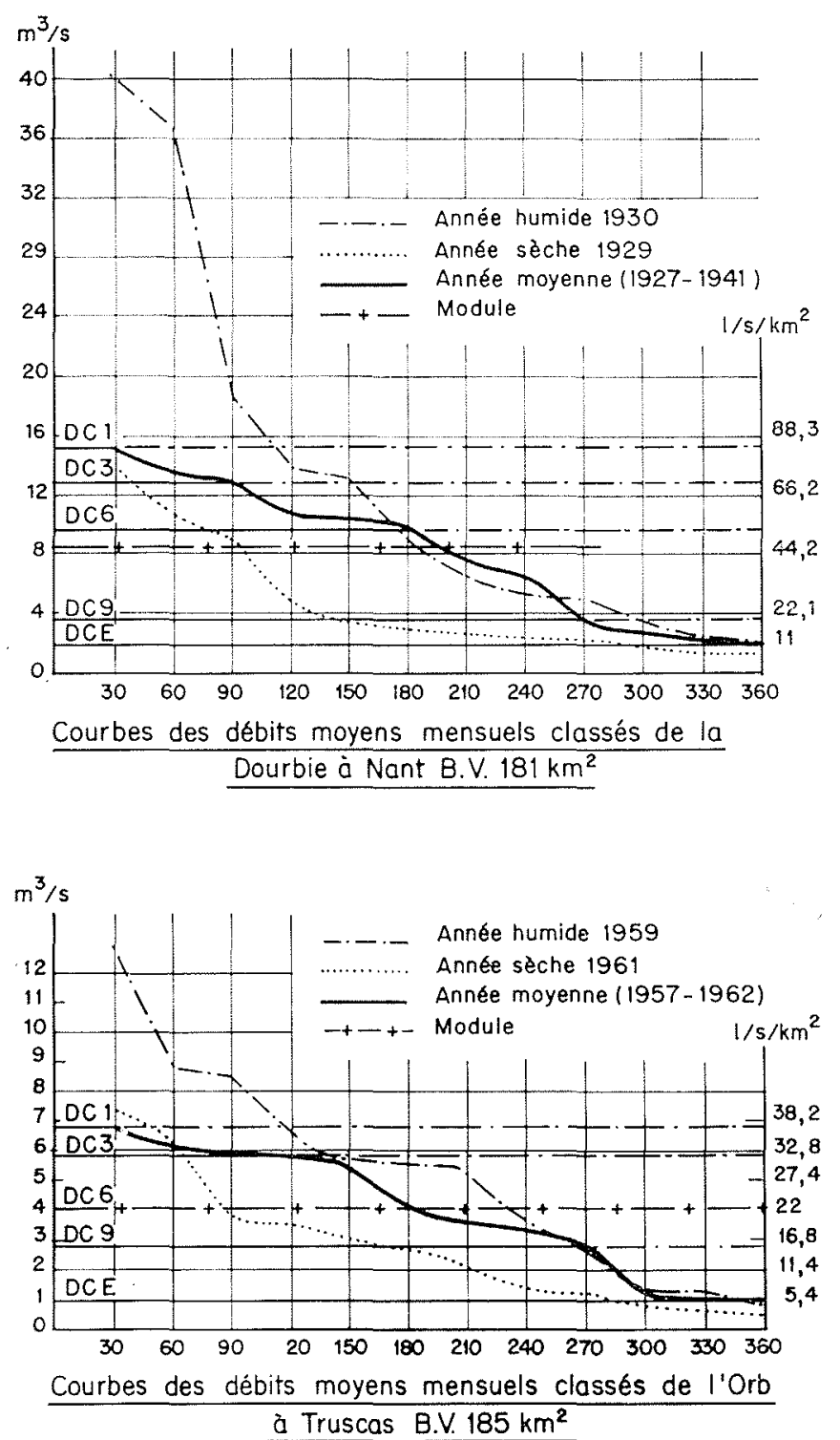

5/

5/ Courbes des débits moyens mensuels classés de la Dourbie (Causse), de l'Orb (Avant-Causse).

6/ Courbe des débits moyens mensuels classés de la Vis (Causse).

7/ Comparaison des coefficients mensuels de débit des rivières du Causse (Dourbie-Vis) et de l'Avant-Causse (Orb-Lergue).

pendant la longue période estivale où elles ne reçoivent aucun appoint.

\section{Hydrogéologie du Karst.}

LES UNITÉS KARSTrQues.

Nous avons distingué deux grandes unités karstiques: d'une part l'Avant-Causse, d'autre part le Causse.

L'Avant-Causse comprend deux niveaux karstifiables: les grès du Trias supérieur et les calcaires de l'Hettangien. De ces deux formations, la première est négligeable et n'offre un intérêt hydrologique qu'au profit de circonstances locales bien précises, en particulier lorsqu'elle se trouve au contact des terrains métamorphiques qui encadrent les Grands Causses (Saint-Léons, Lanuéjols, ...). Les formations

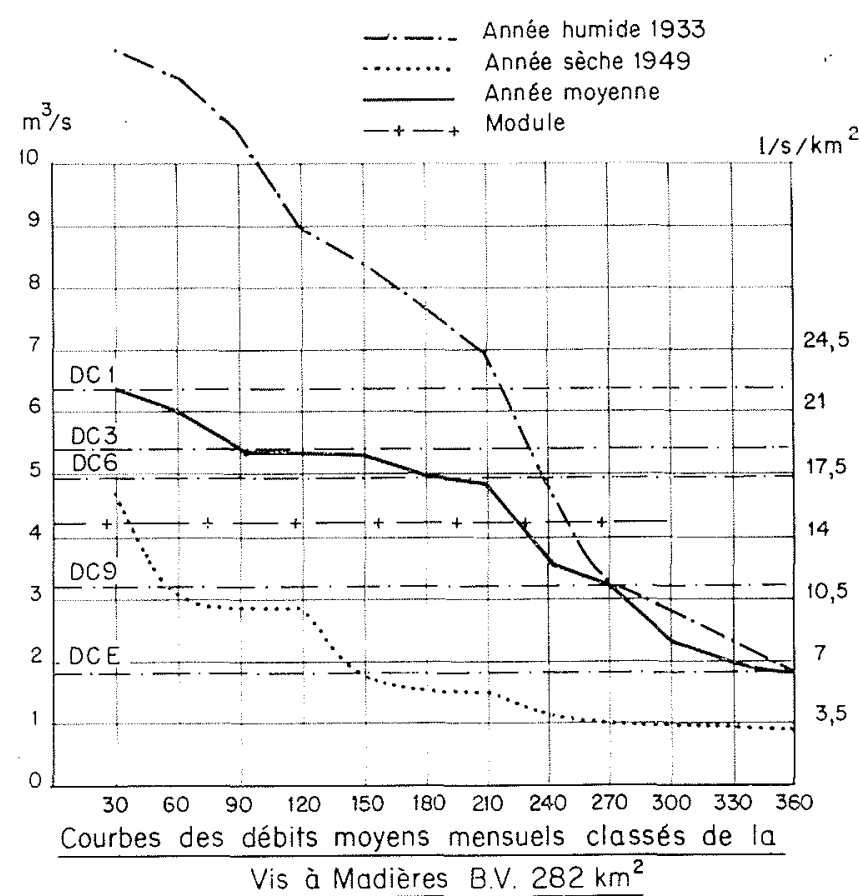

6/

$\mathrm{C} / \mathrm{m} / \mathrm{Q}$

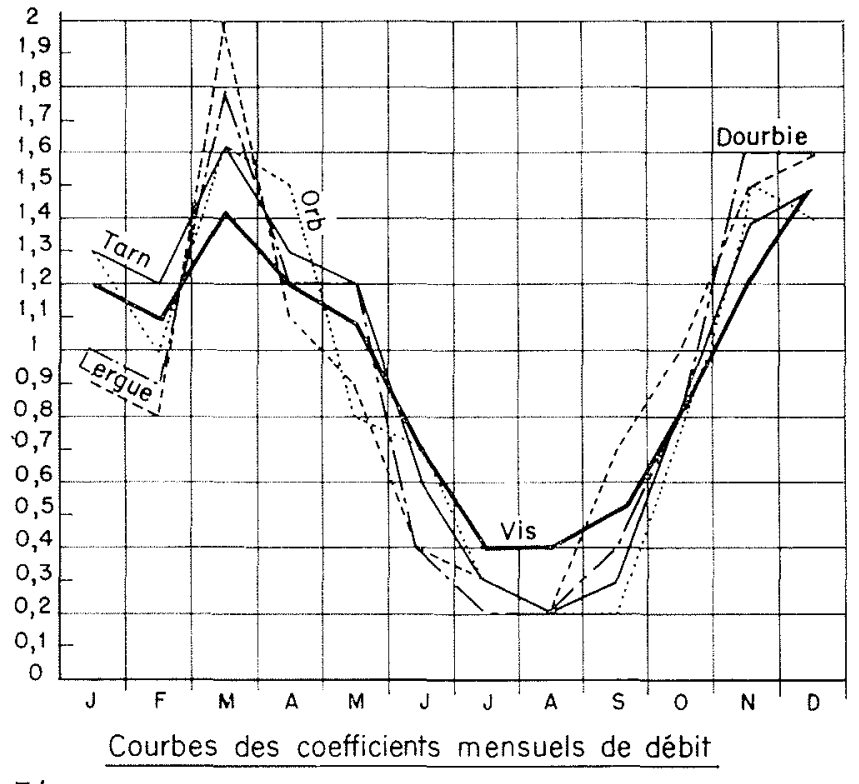

$7 /$

de l'Hettangien abritent des circulations souterraines nettement plus importantes. L'épaisseur karstifiable varie de $150 \mathrm{~m}$ à $200 \mathrm{~m}$ au sud, à quelques dizaines de mètres au nord. Leur composition minéralogique montre que ces calcaires sont riches en dolomite $(92 \%$ à $87 \%)$ et pauvres en calcite ( $4 \%$ et $2 \%$ ).

Le Causse est séparé de l'Avant-Causse par la formation marneuse du Toarcien, parfaitement étanche. Au nord (Millau), elle est épaisse de plusieurs centaines de mètres et se réduit ou est complètement éliminée au sud dans le Lodévois.

La masse karstifiable est représentée par les calcaires du bajocien-bathonien et du jurassique supérieur. La composition minéralogique indique que ces calcaires sont beau- 


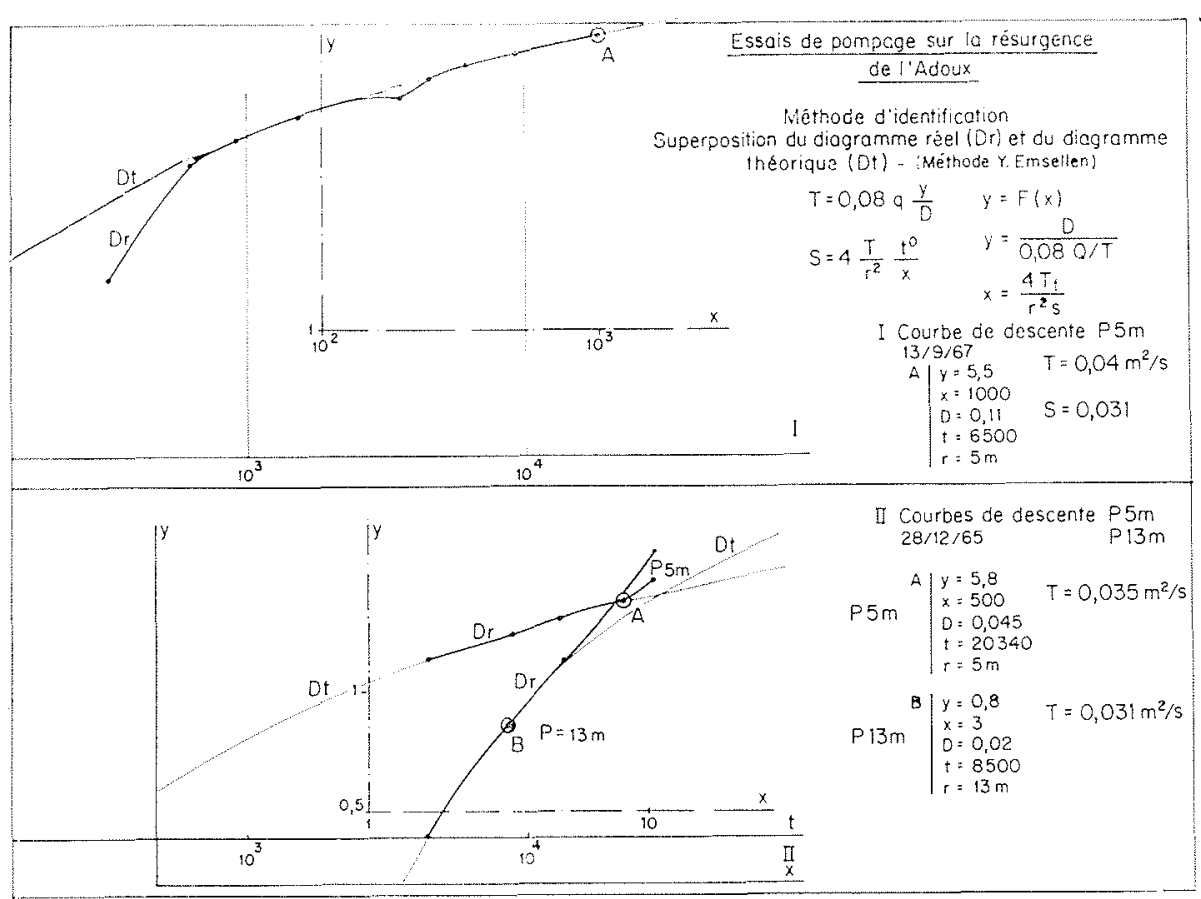

8/

Courbes de descente et de remontée obtenues

lors d'un

essai de pompage sur une nappe de fissures de l'Avant-Causse (Hettangien). coup plus riches en calcite que ceux de l'Avant-Causse :

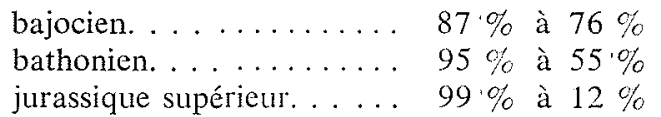

Le pourcentage en dolomite est plus variable. Dans les niveaux les plus dolomitiques (bajocien-bathonien), il varie de $2 \%$ à $90 \%$.

Ce phénomène a une incidence directe sur le mode de circulation des eaux souterraines. L'enrichissement en dolomite des calcaires de l'hettangien diminue en effet leur solubilité, il en résulte une réduction du volume des vides dans l'Avant-Causse et la prédominance des circulations par micro-fissures. Le Causse au contraire est plus largement ouvert aux circulations par canaux et gros conduits.

Les faciès de l'Avant-Causse sont peu influencés par les formes structurales; on note cependant quelques dispositions topographiques qui entraînent une alimentation par pertes (Costes-Gozon, Saint-Léons). Une inclination générale des strates vers l'intérieur du plateau localise les émergences dans les reculées des vallées recoupant l'AvantCausse et favorise l'existence de karsts noyés.

Le Causse est plus affecté que l'Avant-Causse par les dispositions structurales. Les failles réalisent un compartimentage de la masse calcaire en régions hydrologiques autonomes. Des courbures de grande amplitude, qui résultent du jeu des failles, modèlent le toit imperméable et orientent les écoulements profonds (Cernon, Sorgues, Corp, ...).

D'autre part, l'épaississement de faciès hydrologiques favorables (dolomite, calcaire marneux) détermine l'existence de pièges à eau à la surface du Causse (Lanuéjols) et sur sa bordure au niveau des émergences (Espérelle, Durzon).

Nous avons distingué deux systèmes hydrologiques:

- le système hydrologique de l'Avant-Causse;

- le système hydrologique du Causse.

Le système hydrologique de l'Avant-Causse comprend trois sous-systèmes:

- un principal dans l'hettangien;

- deux secondaires au sommet de l'hettangien, dans le trias.

Le système principal de l'hettangien est caractérisé par des circulations dans des diaclases dont la taille varie de quelques mètres à quelques centimètres. L'alimentation est d'origine météorique, rarement par des pertes. Les émergences ont un débit régularisé par l'existence des reienues au-dessus de la « source » ou à proximité, constituant des nappes de fissures (Adoux, La Baume, Aucassie). Les autres systèmes ont une importance moindre, celui du Trias peut abriter des karst noyés non négligeables (Saint-Léons).

Le système hydrologique du niveau bajocien-bathonien inférieur repose sur le niveau imperméable du Toarcien. Il est caractérisé par des ciculations dans de larges réseaux de diaclases très ouvertes. Le bathonien offre toujours des réseaux denticulés où les remplissages de sable sont fréquents. Dans le bajocien au contraire, l'eau circule toujours dans des galeries larges. L'alimentation est météorique, mais aussi assurée par les pertes des principales rivières qui abordent ou traversent le Causse.

Les écoulements se font au niveau du toit imperméable dans la zone ouest du Causse (La Cabane, le Brias, le Boundoulaou), sur le niveau de base local ailleurs (Durzon, Espérelle, Corp,...). Près de la surface, on observe parfois des écoulements pérennes et abondants (bassin de la Sorgues). En hiver, il est fréquent d'y rencontrer des circulations temporaires. Elles peuvent s'inverser et créer des phénomènes d'extravasement.

Lorsque l'alimentation est assurée par des pertes, l'eau emprunte des niveaux privilégiés comme les dolomies. (La Garène, la Jonte, la Dourbie, la Vis.)

Les systèmes secondaires du bathonien supérieur et du jurassique supérieur se caractérisent par l'existence de nappes de fissures situées au-dessus des écoulements par canaux et conduits largement ouverts du système bajocienbathonien.

\section{HYDRODYNAMIQUE DU KARST.}

\section{Action des pompages sur le karst.}

Au cours de notre prospection hydrologique, nous avons effectué des essais de pompage sur des nappes de fissures ou des karst noyés et appliqué à ces essais les méthodes de résolution graphique; dans tous les cas nous avons constaté que les rabattements obtenus en fonction du temps s'alignent convenablement et permettent de calculer des valeurs ap- 
prochées de la transmissivité ( $T$ ), du coefficient d'emmagasinement (S) et de la perméabilité (K) (fig. 8 et 9).

Pour l'ensemble des essais, la valeur de $T$ est comprise entre $\mathrm{T}=\mathrm{n} \cdot 10^{-2}$ et $\mathrm{T}=\mathrm{n} \cdot 10^{-3}$.

Dans les calcaires en plaquettes à double perméabilité horizontale et verticale, elle est de lordre $: T=n \cdot 10^{-3}$ à $\mathrm{n} \cdot 10^{-2}$.

Dans les dolomies, elle est de : $\mathrm{T}=\mathrm{n} \cdot 10^{-3}$.

Dans les calcaires à gros bancs, on $\mathrm{a}: \mathrm{T}=\mathrm{n} \cdot 10^{-2}$

Le coefficient d'emmagasinement (S) varie de $72 \%$ à $1,9 \%$; dans les dolomies la valeur est la plus élevée : de $33 \%$ à $72 \%$. Dans les calcaires marneux, ces valeurs sont faibles: $5,4 \%$ à $1,9 \%$.

La perméabilité $(\mathrm{K})$ varie de $3,3 \mathrm{~cm} / \mathrm{s}$ à $0,1 \mathrm{~cm} / \mathrm{s}$. Dans les dolomies, elle est toujours inférieure à $1 \mathrm{~cm} / \mathrm{s}$. Dans les calcaires très fissurés et à circulation par canaux, elle est de $1,1 \mathrm{~cm} / \mathrm{s}$.

Il semble donc que l'on puisse appliquer aux calcaires les équations de Theis et que des essais de pompage sur les karsts noyés peuvent fournir d'importantes données sur le milieu et les réserves.

\section{Analyse des courbes de tarissement.}

L'étude comparée des hydrogrammes de tarissement enregistrés aux résurgences de la Sorgues, de la Vis et de l'Espérelle nous a permis de déterminer le volume des eaux transité par les gros conduits, celui des eaux provenant de la vidange des chenaux de dissolution, et celui des eaux provenant du ressuyage des fissures ou des interstices.

L'étude des hydrogrammes nous a montré que l'on ne peut pas, toujours, différencier les deux premières phases du tarissement $\alpha_{1}$ et $\alpha_{2}$.

La comparaison des coefficients des composantes du tarissement nous montre les analogies et les différences suivantes :

- pour la Sorgues, l'Espérelle et la Vis, on a :

$2>\alpha_{1}>0,28$

- pour la Sorgues ế l'Espérelle :

$\alpha_{2}=\mathrm{n} \cdot 10^{-1}$ et $\alpha_{3}=\mathrm{n} \cdot 10^{-2}$;
- pour la Vis:

$$
\alpha_{2}=\mathrm{n} \cdot 10^{-2} \text { et } \alpha_{3}=\mathrm{n} \cdot 10^{-3} \text {. }
$$

Le volume le plus important écoulé provient toujours des microfissures. Dans le cas ò̀ elles dominent, comme à la Vis, il est de $90 \%$ à $85 \%$; dans les autres cas de $70 \%$ à $60 \%$.

Par l'étude des courbes de tarissement, on peut différencier les formes d'écoulement $\mathfrak{e t}$ distinguer les réseaux où les circulations sont dominées par les microfissures. D'autre part, cette étude apparaît particulièrement intéressante pour évaluer le volume disponible à plus ou moins longue échéance.

\section{Importance des facteurs lithologiques et structuraux.}

Parmi les formations lithologiques, la dolomie intervient dans l'hydrologie comme un niveau privilégié qui peut jouer le rôle d'un conducteur dans le cas des percées hydrogéologiques ou bien s'avérer comme un niveau imperméable (formation des lacs d'accumulation). Les niveaux marnocalcaires et les grès apparaissent eux aussi avec des caractères particulièrement intéressants, dans l'hydrologie souterraine, en tant que niveau imperméable ou roche magasin.

La recherche de ces niveaux privilégiés devra précéder toute étude hydrologique de type karstique.

\section{Recherche du bilan.}

Nous l'avons abordé de trois façons.

a) En posant l'équation du bilan hydrologique en milieu calcaire définie par H. Schoeller :

$$
\mathrm{P}+\mathrm{Q}^{\prime} r+\Delta^{\prime} s=\mathrm{Etr}+\mathrm{Q} r+\mathrm{Q} n
$$

dans laquelle la somme des entrées se situe à gauche avec $\mathrm{P}=$ pluie; $\mathrm{Q}^{\prime} r=$ eau provenant de l'infiltration des rivières (pertes); $\Delta^{\prime} s=$ eau provenant des variations de volume des réserves. La somme des sorties est placée à droite avec $\mathrm{Etr}=$ évapotranspiration réelle; $\mathrm{Q} r=$ ruissellement vrai; $\mathrm{Q} n=$ débit total sortant aux sources $=\mathrm{I}$.

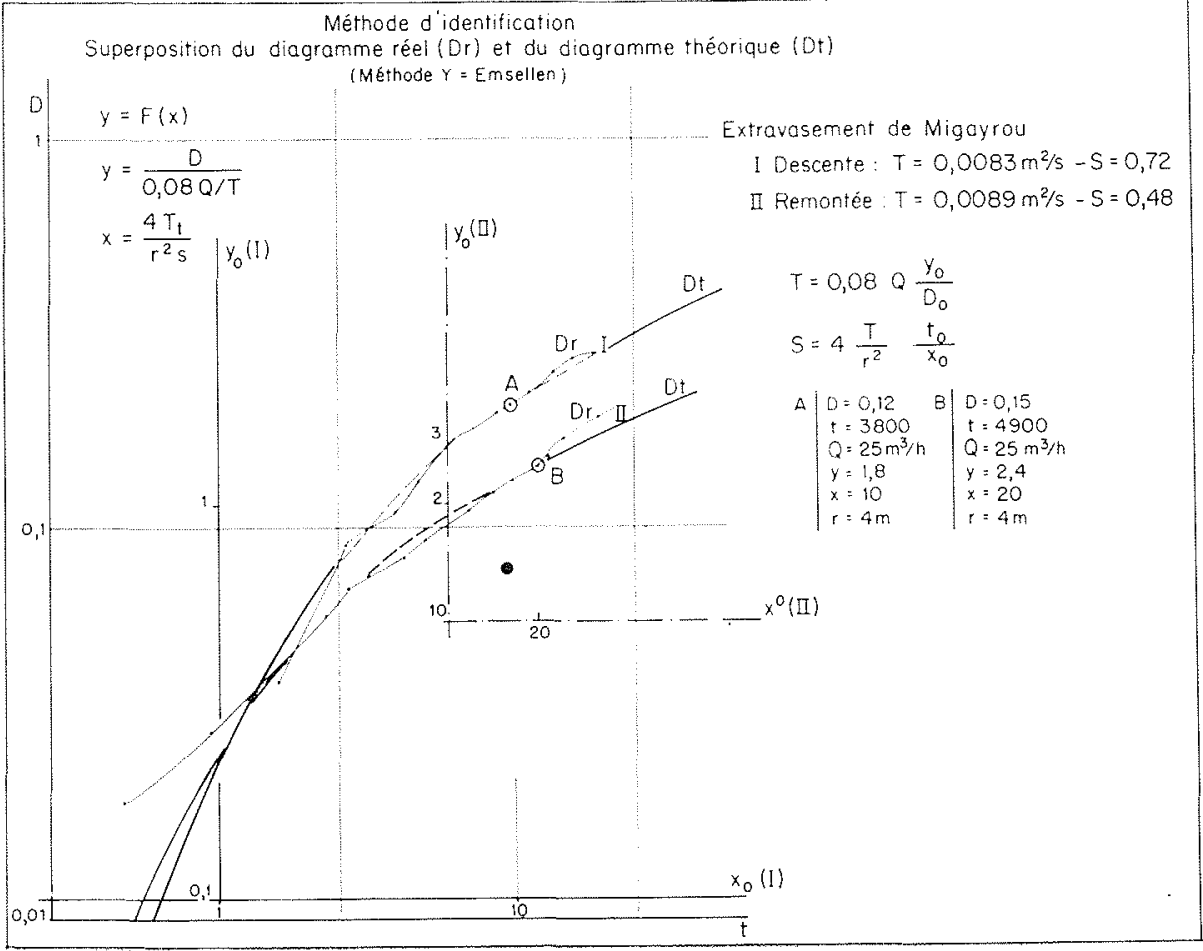




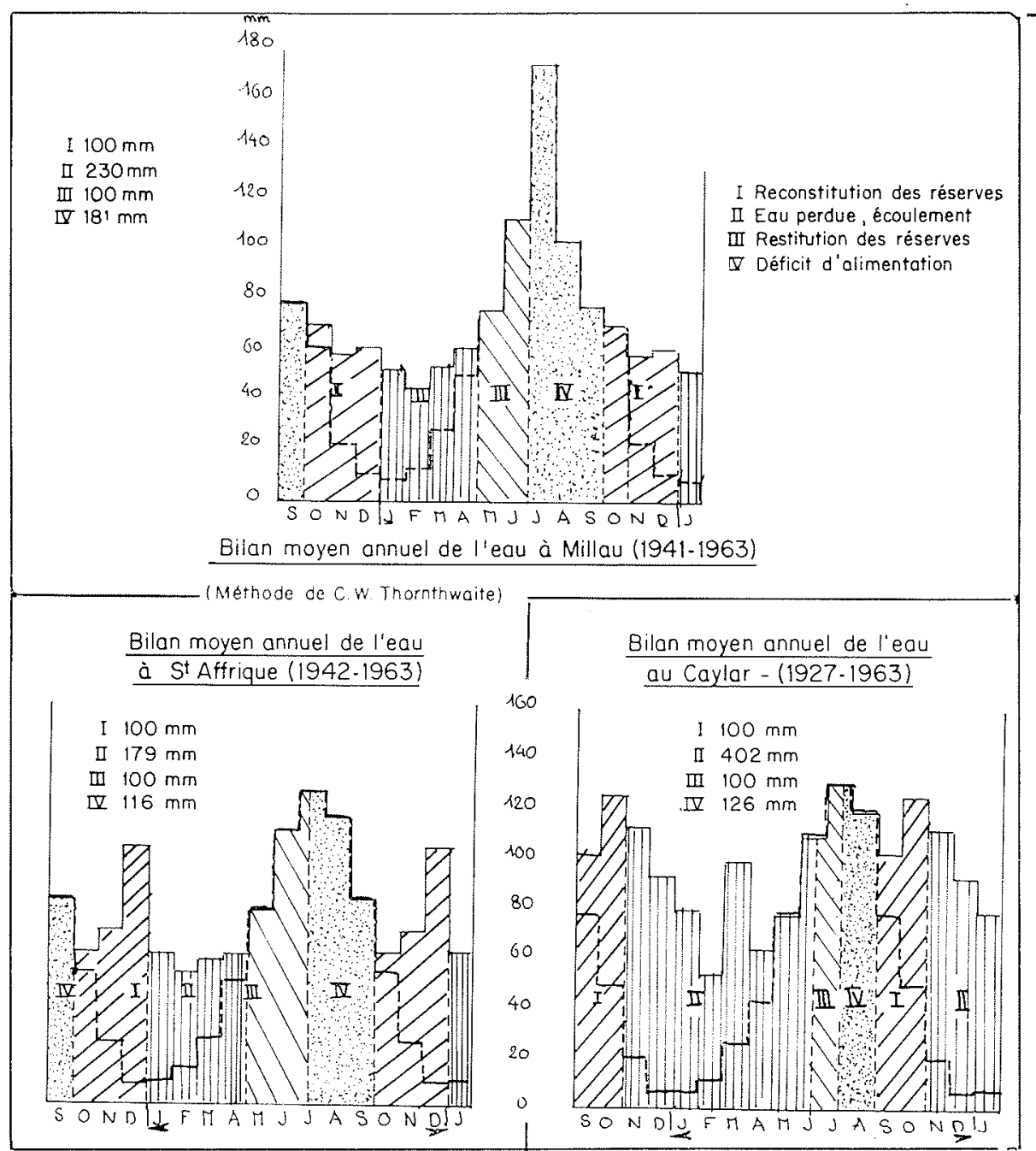

$10 /$

Représentation du bilan moyen annuel de l'eau

pour quelques stations

du Causse suivant

la méthode de Thornthwaite.
Cartes de la répartition mensuelle de la teneur en chlore de l'eau de pluie pour le calcul du bilan stivant la méthode de $H$. Schoeller.
Nous avons établi le bilan pour plusieurs réseaux hydrologiques en utilisant pour Etr les valeurs fournies par la formule de Turc ou de Thornthwaite et en calculant I en fonction des sources: If $(\mathrm{Q})$.

Nous avons ainsi obtenu des coefficients d'infiltration de :

- $10 \%$ à $28 \%$ pour l'Avant-Causse;

- $24 \%$ à $60 \%$ pour le Causse.

b) La recherche du bilan par la méthode de Thornthwaite nous a permis de comprendre comment s'effectue le cycle hydrologique annuel (fig. 10). Nous retiendrons que le déficit d'alimentation varie de $180 \mathrm{~mm}$ à 0 . Il a sa valeur maximale à Millau (181 mm), son minimum à l'Aigoual $(0 \mathrm{~mm})$. Il est de $126 \mathrm{~mm}$ pour le Causse où cette phase dure 1 mois et demi contre 3 mois dans les vallées. Il existe un décalage dans la reconstitution des réserves entre le sud et le nord.

c) L'établissement du bilan par la méthode du chlore (H. Schoeller) nous a montré les variations importantes des concentrations en chlore de l'eau de pluie pour une région déterminée et une même période (fig. 11). Cette remarque entraîne la nécessité de bien connaitre les limites du bassin d'alimentation de l'émergence dont on veut déterminer le bilan. Nous avons obtenu par cette méthode les coefficients d'alimentation suivants :

grès permiens: $15,5 \%$ calcaire : $66 \%$ à $30 \%$.

Ces valeurs sont proches de celles de l'infiltration obtenues par la réalisation du bilan classique. Cette méthode paraît donc convenir à la recherche du bilan en milieu calcaire par sa commodité d'utilisation.

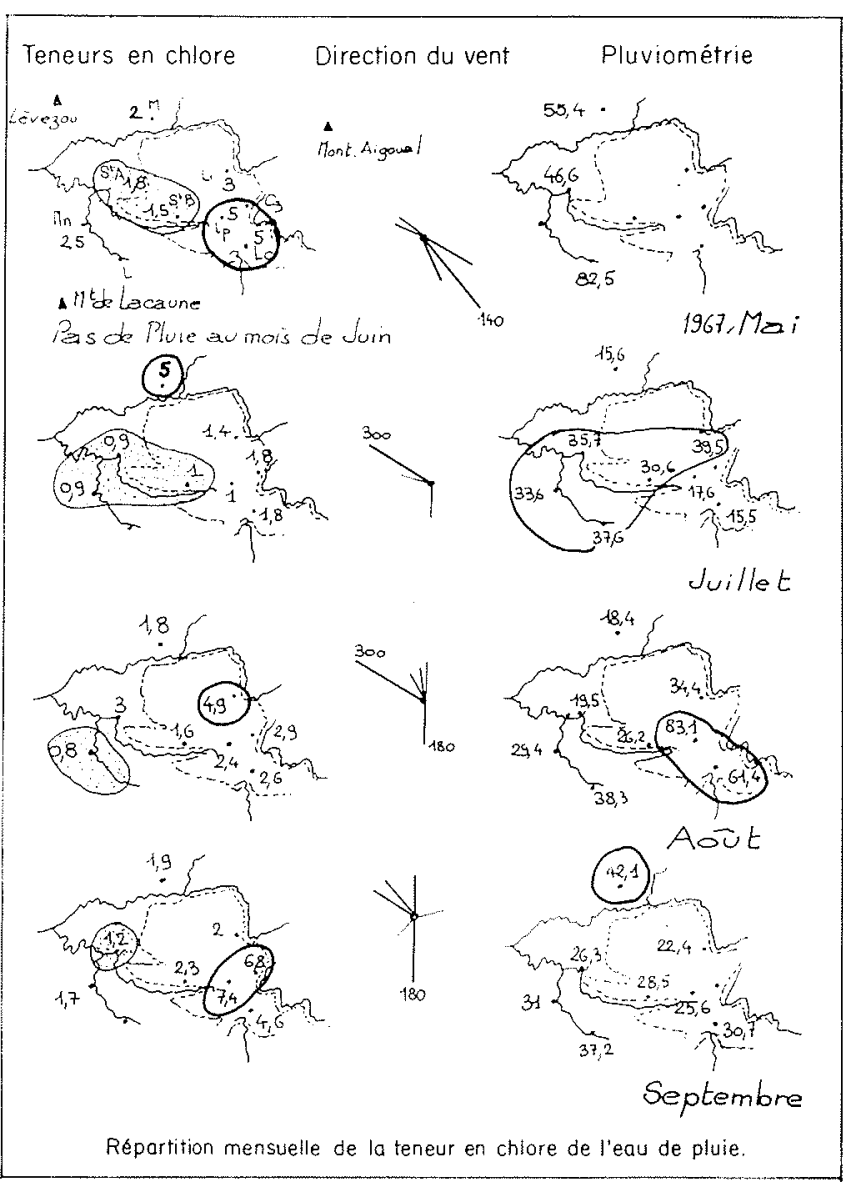


$12 /$

Carte des inondations temporaires du Larzac.

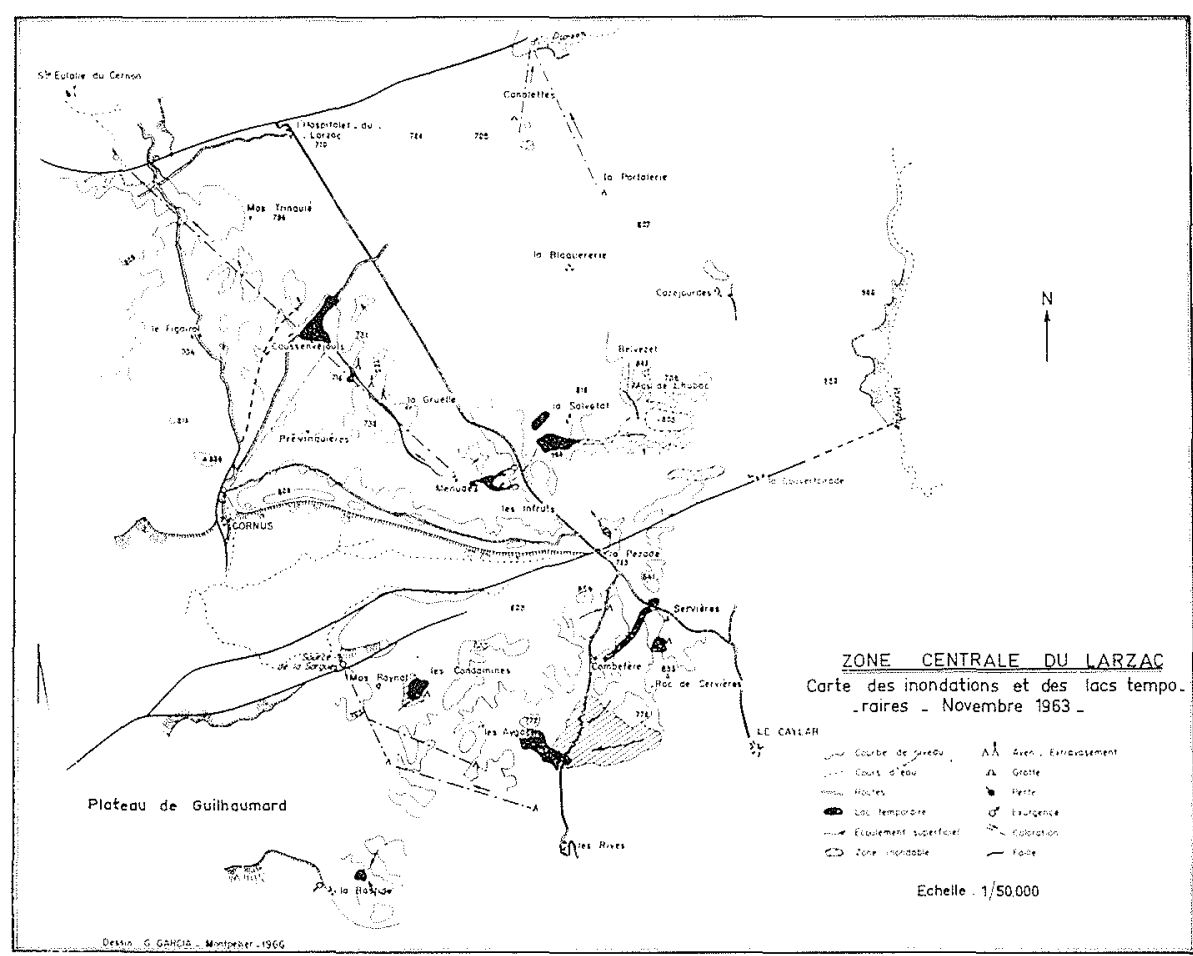

Vitesses d'écoulement.

Trente colorations nous donnent quelques valeurs sur les vitesses des circulations souterraines.

Pour le Causse, on peut admettre $65 \mathrm{~m} / \mathrm{h}$. Elle peut at̂teindre dans des réseaux largement ouverts: $70 \mathrm{~m} / \mathrm{h}$ à l'étiage et dépasser $150 \mathrm{~m} / \mathrm{h}$ lors des crues; dans la dolomie, la vitesse paraît être de $40 \mathrm{~m} / \mathrm{h}$.

Pour l'Avant-Causse, on peut prendre $35 \mathrm{~m} / \mathrm{h}$ comme vitesse moyenne.

\section{Volume des vides.}

L'exploration spéléologique nous a fourni des renseignements sur le développement des réseaux et le volume du cavernement. Pour le Causse, le rapport des vides à la masse karstifiable varie de 1/7000 dans le bassin de la Sorgues à $1 / 400$ sur la bordure ouest. Dans l'AvantCausse, il varie de $1 / 70000$ à $1 / 200$ (valeur qui représente le cas tout à fait exceptionnel des percées hydrologiques).

\section{Phénomènes d'extravasement.}

Nous avons étudié à part les phénomènes d'extravasement et d'accumulation d'eaux liés aux réseaux du LèbreCernon et de la Sorgues (fig. 12).

L'étude détaillée des phénomènes qui déterminèrent les inondations du Larzac en novembre 1963, nous a permis
131

Répartition chimique des eaux souterraines du Causse et de l'Avant-Causse.

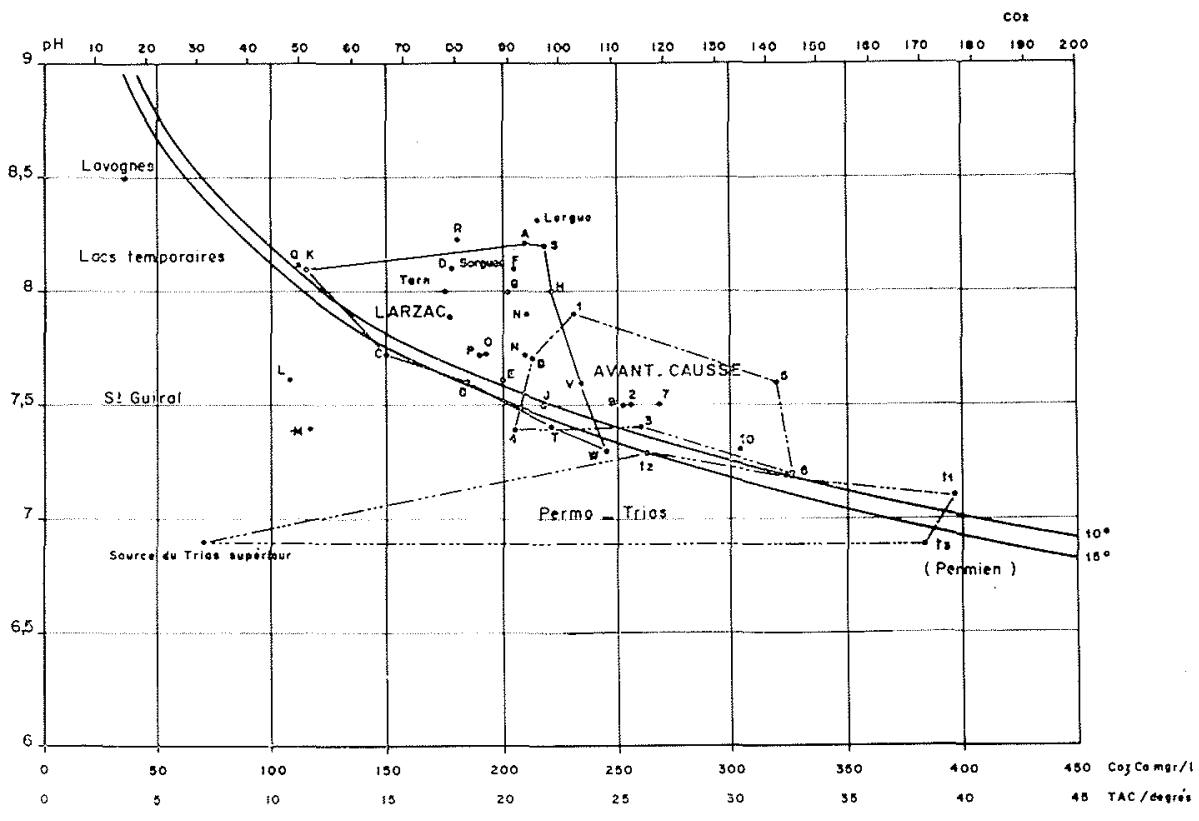

Répartition des equx sur le Diagramme d’Equilibre pH _ TAC.

Courbes déquilibre de TILLMANS (LM.N. Réseau de la Vis), (R.S.T.E.P. Réseau du Cernon) 


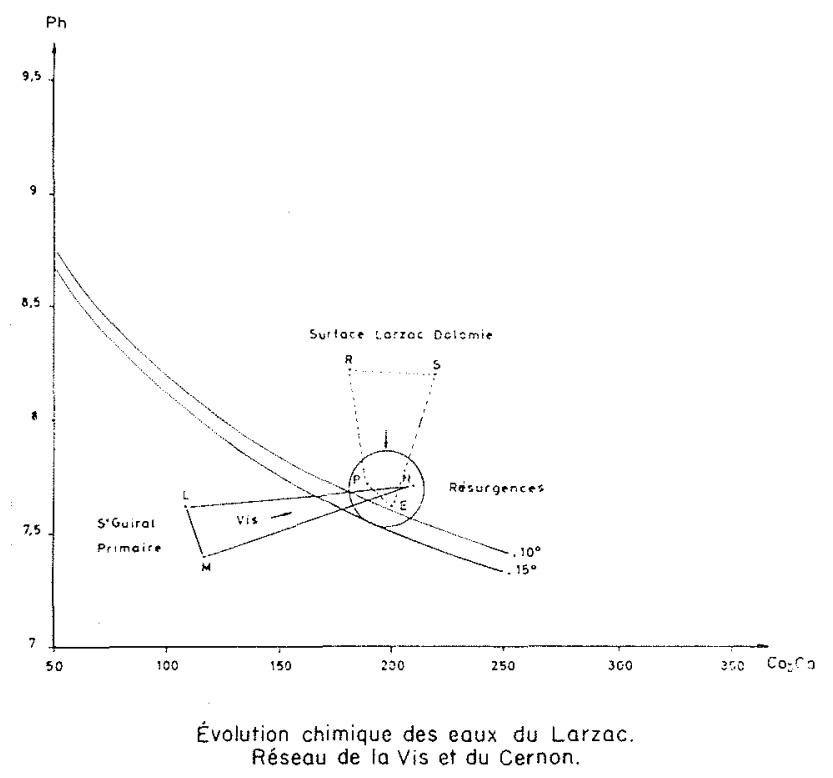

14/ Evolution chimique des eaux qui participent à l'alimentation des résurgences du Causse.

de reconnaître deux catégories de lacs temporaires sur le Larzac :

- d'une part, des lacs d'accumulation dus au rassemblement de l'eau de ruissellement, dans les bas-fonds imperméables dolomitiques;

- d'autre part, des phénomènes d'extravasement liés à des inversions souterraines de l'écoulement et à la réapparition de l'eau dans les bas-fonds dolomitiques du plateau au profit des avens.

L'étude de l'hydrologie souterraine nous apporte deux groupes de résultats :

$1^{\circ}$ Des données d'ordre pratique sur la localisation et l'importance des réserves en eau dans les Causses.

$2^{\circ}$ Des valeurs chiffrées et d'ordre général sur l'hydro. dynamique dans le karst.

\section{Hydrochimie.}

Sur le plan de la chimie des eaux, nous avons tout d'abord procédé à une campagne de mesures physico-chimiques, résistivités et $\mathrm{pH}$ qui a permis de différencier les bassins drainants, de localiser parfois des apports souterrains cachés.
Pour l'ensemble de la région étudiée, la résistivité ramenée à la notion d'indice de solubilité varie dans des proportions importantes. Nous avons noté les valeurs suivantes de l'indice de solubilité (Ims)

- écoulements superficiels et lacs (lavognes). . . . . . . . . . . . . . . .

- écoulements souterrains dans les calcaires du Jurassique moyen............... 32 Ims 39 - écoulements souterrains dans les dolomies. 20 Ims 80

Pour l'Avant-Causse, les pH se situent entre 7,2 et 7,9 ; pour les eaux du Causse 7,5 et 7,7.

L'analyse au laboratoire a montré que les eaux étaient d'une façon générale du type bicarbonaté calcique, mais qu'elles présentaient de nombreuses individualités locales.

Dans l'Avant-Causse, nous avons pu distinguer des eaux bicarbonatées calciques magnésiennes, calciques magnésiennes sulfatées, sulfatées calciques, sulfatées sodiques.

Dans les Causses, elles sont typiquement bicarbonatées calciques, mais se singularisent dans les dolomies oì elles évoluent vers un type calcique magnésien sulfaté.

La répartition des eaux sur les diagrammes de Tillmans montre que les eaux sont en équilibre et leur répartition sur le diagramme suit l'échelle stratigraphique (fig. 13).

Au cours de la traversée des réseaux karstiques et quelle que soit leur origine, les eaux souterraines tendent vers une forme générale du genre $\mathrm{pH}=7,7 ; 20^{\circ} \mathrm{TAC} 21^{\circ}$ (fig. 14)

La comparaison avec les diagrammes d'équilibre des karsts du Languedoc, Provence, Quercy, semble indiquer que l'évolution karstique de la région méridionale des Grands Causses, est stationnaire si ce n'est achevée.

\section{Conclusion.}

Nos recherches nous ont donc permis de mener parallèlement: une étude du cycle hydrologique dans les massifs calcaires de la région méridionale des Grands Causses et une recherche expérimentale sur le déplacement de l'eau dans le milieu karstique proprement dit.

Nous avons analysé chacune des phases de ce cycle en utilisant les méthodes classiques de la climatologie, de la potamologie, de l'hydrogéologie et de l'hydrochimie et nous avons montré comment l'interpénétration de ces différentes disciplines aboutit à une meilleure connaissance des phénomènes hydrologiques. Nous avons souligné d'autre part les techniques d'investigation qui nous paraissent les plus appropriées à la compréhension des circulations souterraines en milieu calcaire. 